\title{
Spiegare le leggi razziali ai bambini
}

\author{
di Elena Mastretta
}

Giu 5, 2019 | Didattica in classe, In evidenza $|\underline{0}|$

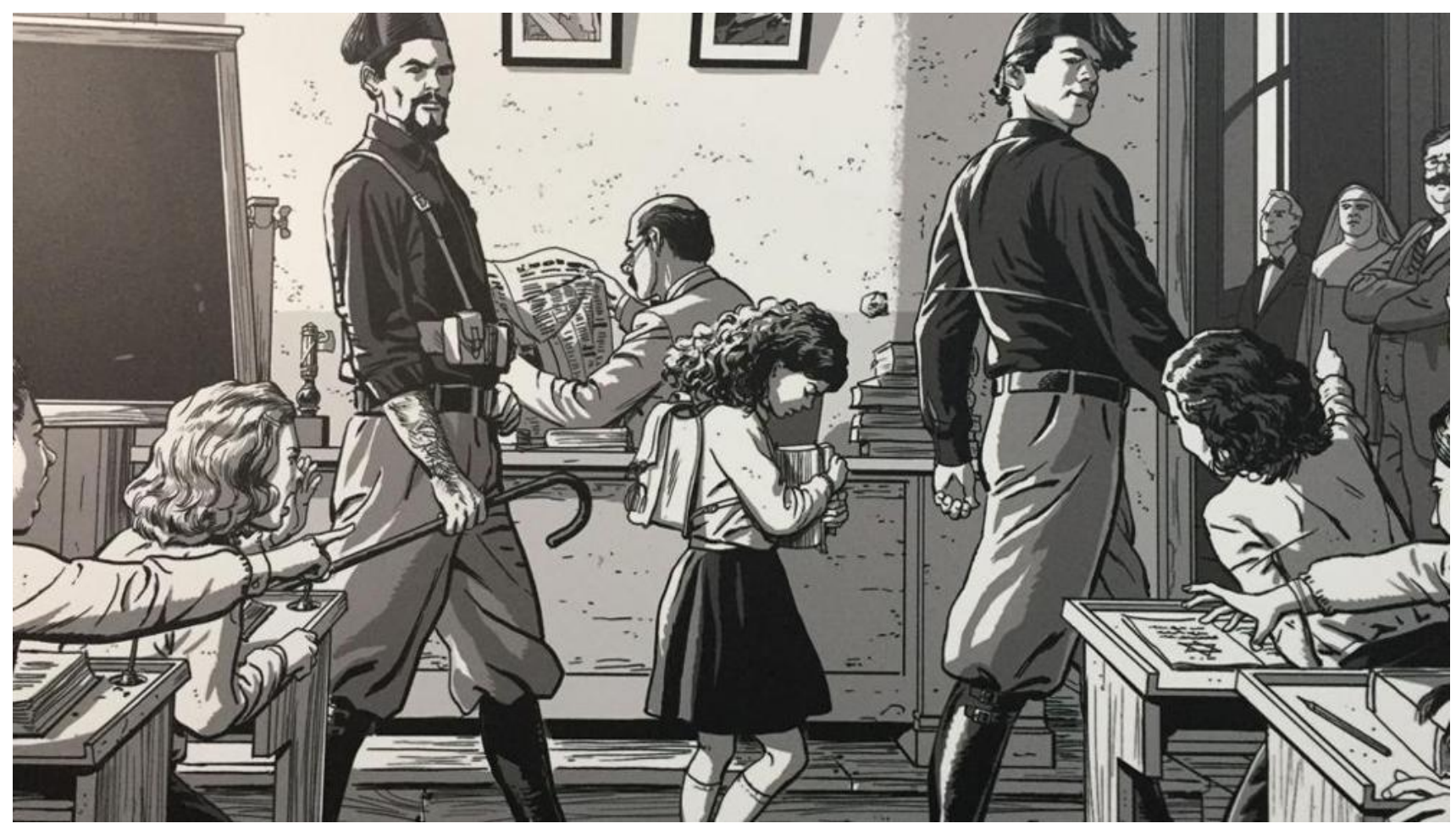

Fumetto di Roberto Zaghi sulle leggi razziali e l'espulsione dei bambini ebrei dalle scuole italiane, tratta da La Stampa, versione on line, 13 aprile 2018, Licenza CC 4.0

\begin{abstract}
In questo articolo viene illustrata un'attività rivolta a studenti della scuola primaria sul tema delle leggi razziali italiane. Il percorso prevede la presentazione di alcune biografie di persone nate tra il 1928 e il 1938 che a diverso titolo e in diversi luoghi hanno subito discriminazione e persecuzione. Le biografie sono state ricostruite a partire da attività di testimonianza dei protagonisti, oppure attraverso $i$ testi che loro stessi hanno prodotto sull'esperienza, o, ancora, tramite documenti d'archivio.

\section{Parlare di "tematiche difficili" alla primaria}

Il tema della memoria, ormai prepotentemente entrato nella didattica in ogni ordine e grado di scuola, incontra per le sue caratteristiche alcune difficoltà di trattazione alla scuola primaria. Seguendo le indicazioni didattiche di Yad Vashem, può essere utile con i più piccoli soffermarsi sui concetti di emarginazione, persecuzione e discriminazione senza addentrarsi nella descrizione della macchina dello sterminio, anche in considerazione del fatto che sono davvero poche le biografie di bambini che hanno affrontato l'internamento e che possono avere una narrazione, dal momento che i bambini erano di norma gassati al loro arrivo nei centri di messa a morte immediata.
\end{abstract}


Le biografie di coloro che, essendo arrivati nel campo molto giovani, sono sopravvissuti al sistema concentrazionario sono di certo molto interessanti, ma siamo spesso di fronte a preadolescenti, piuttosto che a bambini. Se si pensa di voler affrontare anche con allievi molto piccoli il tema dell'internamento e della vita nel campo, il caso di Liliana Segre, della quale sono disponibili in rete molti filmati[1] di testimonianza e che ha scritto diversi libri adatti anche ad un giovane pubblico[2] può essere un buon punto di partenza, che troviamo molto interessante accostare, nella presentazione, a quella di Bogdan Bartnikowski[3], autore del testo Infanzia dietro il filo spinato, che negli ultimi tre anni ha testimoniato la sua esperienza nel campo di Auschwitz alle scolaresche e alla cittadinanza di Novara.

Mentre Liliana arriva ad Auschwitz come deportata razziale e lavora come schiava al servizio del Reich, Bodgan e gli altri ragazzi polacchi di cui ci parla sono stati arrestati durante l'insurrezione di Varsavia e, pur vivendo nelle baracche sottoposti al caldo, al freddo e alla privazione del cibo, non sono costretti al lavoro coatto se non nell'ultima fase della prigionia. La presentazione di queste due biografie permette di far comprendere che i campi non sono destinati esclusivamente agli ebrei, ma che sono molte le persone che il nazifascismo vuole eliminare[4].

Attraverso il raffronto si possono invitare i ragazzi a cogliere somiglianze e differenze sul trattamento che ebrei e non ebrei subivano, su come reagivano. Ad esempio, affidando ai ragazzi una selezione di brani sui due casi, l'insegnante può stendere un elenco di "caratteristiche" della prigionia da confrontare sulla base di una tabella. Lo stesso lavoro può essere fatto a partire da una ricerca, da una narrazione dell'insegnante, visionando due filmati tra quelli disponibili in rete.

\section{Non solo sterminio}

L'identificazione in queste storie da parte dei bambini della primaria è comunque difficile, perché si tratta di esperienze estreme, che essi non riescono a ricollocare nell'orizzonte della loro quotidianità. Riteniamo sia importante ricordare ai più piccoli che il progetto di distruzione totale non ha avuto successo e che almeno alcuni di coloro che il nazifascismo voleva sterminare si sono salvati. Affrontando le biografie di salvati, intendendo con questo termine non solo reduci e sopravvissuti dei campi, ma anche coloro che riuscirono a evitare la cattura venendo comunque sottoposti alle vessazioni legislative, cerchiamo di non ridurre la storia della shoah alla sola storia del meccanismo di sterminio. Come in un libro di mille pagine, questo capitolo arriva in fondo ed è giusto affrontarlo quando si avranno gli strumenti (anche in relazione all'età) per comprenderlo.

\section{La persecuzione dei bambini}

Con chi ha meno di 12-13 anni, secondo la nostra esperienza, la comprensione del fenomeno persecutorio è immediata a partire da biografie di "vittime delle leggi razziali": bambini che sono stati cacciati da scuola, esclusi dai gruppi di amici, che hanno vissuto il licenziamento dei genitori, le incertezze e le difficoltà del trasferimento, del cambio di nome e di identità. Ancora di più, che hanno vissuto isolati dal mondo, anche lontani dal resto della famiglia, se sono stati dei "bambini nascosti”[5]. Queste storie possono essere ancora ascoltate dalla diretta voce dei protagonisti, che essendo molto piccoli durante la seconda guerra mondiale, sebbene anziani, sono ancora in vita. Alcuni di loro hanno inoltre scritto, in genere rivolgendosi ad un pubblico giovane, testi sulla loro esperienza di esclusione e persecuzione e questi possono essere letti nella loro interezza se si affronta una sola biografia, magari per arrivare ad un incontro diretto, oppure essere esaminati per confronto sui temi ricorrenti.

Si tratta di una testimonianza molto particolare, diversa da quella di coloro che sono sopravvissuti al sistema concentrazionario. Sono storie di individui che, giovanissimi, hanno subito forti traumi e le cui famiglie possono essere state sterminate oppure no, ma che hanno avuto a causa di queste 
vicende sempre gravi ripercussioni sulla loro vita dopo la fine della guerra. La loro testimonianza è più tardiva di quella dei sopravvissuti dei campi, essi stessi sentono dall'inizio una differenza tra ciò che hanno subito e ciò che sentono raccontare da altri che non sono riusciti a sfuggire alla cattura e alla deportazione. A volte, la loro testimonianza è stata impedita anche dall'assenza di memoria del periodo nascosto, attribuibile all'età e al trauma subito. Esistono quindi numerosi elementi di identificazione tra loro all'epoca dei fatti e gli studenti più giovani.

In particolare, la lettura comparata dei testi Il giorno che cambiò la mia vita di Cesare Moisé Finzi e Una bambina e basta di Lia Levi si presta bene a raccontare la volontà persecutoria delle leggi razziali italiane e la loro pervasività. Si tratta di un percorso di lettura guidata che può essere svolto come attività a sé stante, ma che nel nostro caso è spesso utilizzato come propedeutico all'incontro con un testimone delle leggi razziali, Franco Debenedetti.

\section{Bambini nati senza diritti}

Desideriamo qui condividere l'esperienza pluriennale di proposta alle classi della scuola primaria della testimonianza di Franco Debenedetti. Franco è un testimone delle leggi razziali, un bambino nascosto, che da quando ha iniziato a ricostruire la sua biografia infantile testimonia le conseguenze della persecuzione razziale sulla sua vita e su quella dei suoi familiari. Lo fa attraverso incontri strutturati per livelli di età che conduce ormai da oltre dieci anni in tutta Italia e all'estero avvalendosi di supporti multimediali e di una ricca documentazione originale, parte della quale è confluita in una mostra da lui realizzata nel 2008 per le Biblioteche Civiche Torinesi e la cui esposizione può essere abbinata alla testimonianza[6].

L'opportunità di incontro diretto con i bambini, dopo la verifica della metodologia di testimonianza adottata, è stato l'elemento che ci ha portato a proporre alle scuole primarie che ci chiedono interventi sul tema della memoria di far incontrare le classi con Franco. Con i bambini più piccoli è il Franco bambino che racconta, creando un clima di ascolto ed attenzione che permette ai presenti di riconoscere nelle dinamiche persecutorie e di esclusione descritte meccanismi conosciuti e di smascherarli/identificarli.

\section{La preparazione all'incontro}

Di norma, prima che i ragazzi incontrino il testimone, noi incontriamo i docenti per un approfondimento sul tema delle leggi razziali italiane, la cui conoscenza deve essere trasmessa dagli insegnanti ai bambini in quanto tutta la testimonianza parte dalla loro entrata in vigore. Per farlo, non volendo anticipare i contenuti della testimonianza se non attraverso la documentazione che il testimone stesso invia agli insegnanti, utilizziamo una serie di immagini e una antologia di testi di altri bambini, quasi sempre italiani, che hanno provato la persecuzione razziale e che l'hanno successivamente raccontata, partendo dai testi di Finzi e Levi che abbiamo precedentemente citato, ma moltiplicando i casi per dare l'idea dell'ingresso/influenza capillare delle leggi razziali nella comunità ebraica italiana.

\section{Chi sono le vere vittime?}

A lungo si è ritenuto che "essere vittima delle leggi razziali" fosse un'esperienza di secondo livello rispetto alle vittime della shoah tradizionalmente intese e che questo tipo di testimonianza non avesse un valore storico rilevante[7]. Oggi le posizioni sul tema sono cambiate e si comincia a dare spazio, anche nella ricerca storica, alla raccolta delle storie dei bambini, in particolare di quelli che vengono chiamati "bambini nascosti". Tuttavia, i testi editi sono ancora un ristretto numero (anche se ci sono autori che ne contano parecchi). Non è questa la sede in cui riflettere sull'opportunità di una raccolta e messa a disposizione sistematica di queste testimonianze, ma l'incontro a scuola 
potrebbe comunque favorire l'avvio di una ricerca, ad esempio, negli archivi scolastici per verificare se ci furono espulsioni tra gli alunni. Questa attività di ricerca nelle scuole piemontesi, in occasione dell'Ottantesimo anniversario dell'entrata in vigore dei provvedimenti in difesa della razza italiana, è stata svolta in numerosi casi, permettendo di riportare alla luce storie di persecuzione dimenticate che si stanno trasformando in concrete azioni di memoria attraverso mostre, rappresentazioni teatrali, intitolazioni.

Questa stessa introduzione sul tema delle leggi razziali condotta attraverso la narrazione di alcune biografie è quella che viene poi proposta agli alunni da noi o dall'insegnante, insieme alla lettura di alcuni testi inviati dal testimone stesso prima dell'incontro di testimonianza, in modo che abbiano le conoscenze utili a comprendere i riferimenti storici della testimonianza.

\section{Raffronto tra diverse biografie}

Anche per mettere in evidenza le peculiarità della storia di Franco, la scelta delle storie di raffronto si focalizza su alcune tematiche ricorrenti: la perdita del lavoro dei genitori, la necessità di trasloco, il clima familiare mutato, l'espulsione da scuola, l'abbandono da parte degli altri e degli amici, il cambio del nome. Infine, le esperienze di vita nascosta vera e propria.

Nel caso di Franco, due sono gli aspetti importanti da sottolineare: ha vissuto tutta l'infanzia sotto la scure delle leggi razziali, non avendo alcuna idea di cosa fossero un clima familiare sereno e rapporti sociali al di fuori della famiglia. Altra particolarità è il modo in cui vive il periodo successivo all'abrogazione delle leggi razziali, aspetto che non sempre viene trattato da altri autori. Lui stesso ne parla ai bambini durante gli incontri di testimonianza, ma già nella fase preparatoria, leggendo i brani che si indicano, si cerca di evidenziare questo tipo di differenze.

\section{L'incontro con il testimone}

Dopo l'Introduzione al tema delle leggi razziali in Italia condotta attraverso la lettura dei brani appositamente selezionati sopra descritti, i bambini incontrano il testimone per una intera mattinata o pomeriggio. Attraverso la proiezione di filmati e documenti, spesso dell'archivio familiare, Franco ricostruisce con i presenti i suoi primi 8 anni di vita. Nato nel dicembre 1937, egli nasce praticamente senza diritti e inizia da subito a subire le conseguenze dei cambiamenti che le leggi razziali introducono nelle vite dei suoi genitori. Il padre, chimico per la Marina, viene licenziato e la famiglia perde l'alloggio che il lavoro del padre, soggetto a continui trasferimenti, garantiva. Le tappe successive sono la partenza verso la Francia di Franco, mamma, papà, del fratello Sergio poco più grande di lui - dei nonni. Il padre fatica a trovare lavoro e sono costretti a "vivere della bontà di chi li ospita"[8], con grandi malumori del nonno che si lamenta spesso per la situazione. Questo crea un continuo disagio e tensione che vengono percepiti da Franco in modo particolare. Del resto, però, il bambino non ha esperienza di periodi di serenità e crede che sia questa la norma. Ben presto bisogna evitare qualunque contatto con le altre persone, quindi la vita dei Debenedetti e i loro contatti umani sono limitatissimi. A Franco e Sergio vengono imposti una serie di divieti che li fanno vivere in un continuo stato di terrore e paura. Non hanno contatti con altre persone o con altri bambini, non frequentano luoghi pubblici, non hanno giocattoli.

L'evolversi delle vicende belliche impongono poi il rientro in Italia e un nuovo peggioramento delle condizioni economiche della famiglia. Franco, che nel frattempo è cresciuto, continua a non poter frequentare bambini, a confrontarsi solo con Sergio, che, essendo più grande, "vince sempre"[9]. Nell'ultima fase della persecuzione al bambino vengono tolti anche lo Shaddai[10] e il nome. 
Tutto questo viene raccontato mostrando gli oggetti, facendo chiari esempi ai bambini, sollecitando la loro attiva partecipazione.

\section{E dopo la guerra?}

La biografia della prima infanzia di Franco Debenedetti si scosta da quella di Levi e Finzi in diversi punti, pur avendo degli aspetti di somiglianza. Ad esempio, Franco non conosce il trauma dell'allontanamento da scuola, perché a scuola non è mai andato. Non sa cosa voglia dire perdere un amico, non avendone mai avuto uno. Il suo mondo per anni conosce come attori solo i familiari e pochi conoscenti, cui non si deve dare confidenza e non esistono momenti di tranquillità, di non allerta. I giorni e le notti scorrono nello sforzo incessante di non commettere azioni sbagliate. Il bambino si sente tranquillo solo in montagna, tra alberi e piccoli animali.

Anche suo padre è stato licenziato, loro sono diventati economicamente deboli, hanno dovuto lasciare la casa e nascondersi. Ma essendo così piccolo, ha provato ciò che hanno provato Cesare e Lia?

Questo senso di non appartenenza non finisce con la fine della guerra: abituarsi a frequentare le persone, a rispettare le regole diviene per il piccolo Debenedetti un esercizio al quale applicarsi con la stessa tenacia con cui in precedenza aveva cercato di diventare trasparente, ma i risultati sono deludenti per sé e per i genitori. Franco si definisce ancora oggi pensando al Franco di allora come "un selvaggio", che dal 1945 in avanti, essendo già grandicello, ha dovuto imparare tutto ciò che per i suoi coetanei sembrava naturale.

\section{Una persecuzione infinita}

Nel soffermarsi a tracciare anche la sua storia dopo la fine della guerra, Franco sottolinea come la memoria della sua infanzia di bambino ebreo perseguitato dalle leggi razziali sia una conquista recente, che ancora oggi è in via di arricchimento. Dopo il 1945 egli ha "dimenticato" gli anni nascosti, i posti dove ha vissuto. Le leggi hanno continuato a perseguitare la sua famiglia anche dopo la loro abrogazione: suo padre e suo fratello pochi anni dopo la fine della guerra si suicidano, in casa non si riesce ad affrontare tutto questo parlandone serenamente, ricostruendo gli anni di persecuzione e fuga. Lui e la mamma conducono una vita apparentemente normale, di riscatto, ma non ha il tempo e la capacità di sciogliere i nodi più grossi del loro dolore.

Solo con il pensionamento Franco si sente pronto ad aprire le cantine delle case ormai vuote per recuperare la memoria di quegli anni e lo fa con un processo lento, faticoso, meticoloso, ma necessariamente non lineare. Anche questo aspetto fa parte della narrazione ai bambini: le guerre non iniziano e non finiscono con una data precisa. Il bambino nato senza diritti è stato un adulto che i diritti conquistati non ha potuto goderli appieno. Anche in questo "finale non lieto" la biografia di Franco si discosta dalle altre presentate.

\section{La riflessione personale dei ragazzi}

Dopo l'incontro Franco lascia alcuni suggerimenti per la creazione di un elaborato che faccia riferimento alla parte della testimonianza che ha più colpito i ragazzi e li invita a realizzare un prodotto. Possono lavorare a piccoli gruppi, ma la richiesta è che l'intervento degli insegnanti sia minimo: non si aspira ad avere un oggetto bello, un disegno perfetto, una canzone ben eseguita o un testo grammaticalmente corretto, ma qualcosa di significativo. 
L'ultima tappa di questo percorso di testimonianza, che viene svolta da una percentuale significativa di classi che aderiscono al percorso, è un successivo incontro con Franco nel quale i ragazzi gli consegnano i lavori svolti. Qualora le classi abbiano lavorato, il testimone si rende disponibile per incontrare di nuovo i ragazzi, che vengono organizzati in fotografo, segretario, regista e uno ad uno, o a piccoli gruppi, presentano a Franco il loro prodotto. Sono inviati a descriverlo in alcune battute (il record vocale viene registrato). I lavori, le foto e le registrazioni audio acquisite da Franco nell'incontro di restituzione vanno ad implementare il suo già ricco archivio di produzioni didattiche. Archivio ordinatissimo e ispezionabile attraverso un dettagliato sistema di catalogazione, che viene utilizzato per produrre mostre delle restituzioni e che è già stato utilizzato da studenti universitari per la scrittura della tesi di laurea. Questa modalità "lunga" di rapporto tra il testimone e gli studenti, che talvolta quando viene illustrata nei primi contatti con la scuola spaventa, è stata costruita e provata nel tempo per rispondere alle critiche che gli incontri di testimonianza talvolta suscitano: i ragazzi si distraggono perché non hanno gli strumenti per capire, l'esperienza resta isolata, ecc. Nel suo complesso questo percorso richiede alle classi circa 5 ore per gli incontri in presenza, due per la preparazione preliminare e un numero variabile di ore, che l'insegnante referente può comunque gestire secondo le proprie esigenze, per la realizzazione dei prodotti dei ragazzi.

\section{La mostra: un'ulteriore possibilità}

Come accennato, nel 2008, in occasione del Settantesimo anniversario dell'emanazioni delle "Leggi razziali italiane" le Biblioteche Civiche Torinesi chiesero a Franco Debenedetti di realizzare una mostra sul tema. La mostra, che da allora è stata esposta per diverse migliaia di giornate in Italia $\mathrm{e}$ all'estero, consta di 18 pannelli, di cui uno di introduzione storica generale che trattano il pregiudizio, la propaganda e che ricostruiscono storie di discriminazione ai danni di ebrei italiani o stranieri residenti in Italia più o meno note. Così troviamo Rita Levi Montalcini, ma anche famiglie le cui vicende di persecuzione sono raccontate dalle foto uscite dagli album di famiglia, dai certificati con il timbro "razza ebraica", dalle lettere di licenziamento.

Consigliamo, ogni volta che è possibile, di esporre la mostra nei locali delle scuole nei giorni precedenti l'arrivo del testimone. Questo permette di proporre l'inquadramento storico già attingendo a materiale che verrà ripreso nella testimonianza. La mostra può poi essere nuovamente visitata dopo l'incontro con Franco, per le domande che necessariamente sorgono spontanee dopo il racconto, ma che ragazzi non sono in grado di porre immediatamente. Può essere utile, anche ai fini del coinvolgimento delle famiglie, oppure in tutte le situazioni in cui episodi di intolleranza e pregiudizio si siano verificati, aprire alla cittadinanza, a partire dalle famiglie degli studenti, la visita alla mostra, magari affidando proprio ai ragazzi il compito di guide.

Proponiamo di seguito alcune schede biografiche dei bambini i cui raccontiamo le storie, tabelle di analisi, una piccola antologia di testi.

\section{Schede bambini}

\section{Lia Levi}

Lia Levi, la cui famiglia è piemontese, vive a Roma, città nella quale lei e i suoi cari si sono trasferiti in seguito alle leggi razziali. Qui ha diretto per trent'anni il mensile ebraico Shalom. Ha pubblicato numerosi libri, quasi sempre ispirati dalla sua vicenda persecutoria, l'ultimo dei quali è Questa sera è già domani, vincitore del Premio Strega Giovani 2018. Nel 2012 le è stato conferito il 
Premio Pardès per la Letteratura Ebraica. Ha pubblicato con diverse case editrici, ricevendo numerosi riconoscimenti. Incontra spesso gi studenti per raccontare la sua vicenda di bambina sotto le leggi razziali, vicenda rispetto alla quale, pur riconoscendo la drammaticità del periodo e il dolore vissuto dalla sua famiglia, riesce a parlare con una certa ironia, merito, afferma, della capacità che ebbero i suoi genitori di gestire la comunicazione con lei in quel periodo.

Bibliografia essenziale di utilizzo per il percorso

- Levi L. 1994, Una bambina e basta, Roma: Edizioni e/o

- Levi L. 1997, Tutti i giorni della tua vita, Roma: Edizioni e/o

- Levi L. 2008 Trilogia della memoria. Tre romanzi all'ombra delle leggi razziali (raccolti in un volume unico pubblicato nella collana Super delle Edizioni E/O)

- Levi L. 2014, Il braccialetto, Roma: Edizioni e/o

- Levi L. 2018, Questa sera è già domani, Roma: Edizioni e/o

- Lievi L. 2003, La lettera B. I sei mesi che hanno sconvolto la mia vita, Milano: Mondadori

- Lievi L. 2003, La Villa del Lago. La Repubblica di Salò 1943-1944, Milano: Mondadori

- Lievi L. 2011, La Villa del Lago. La Repubblica di Salò 1943-1944,Milano, Piemme

- Lievi L. 2005, La ragazza della foto, Milano: Piemme

- Lievi L. 2005, La portinaia Apollonia, Roma: Orecchio Acerbo

- Lievi L. 2006, Un cuore da Leone, Milano: Piemme

- Lievi L. 2008, Il tesoro di Villa Mimosa, Milano: Piemme

- Lievi L. 2010, Siamo in guerra e nessuno ce lo dice, Milano: Mondadori

\section{Cesare Moise Finzi}

Cesare Finzi nasce a Ferrara nel 1930. Cardiologo, è autore di alcune pubblicazioni scientifiche di argomento ebraico e del volume uscito nel 2006, presso Il ponte Vecchio, Qualcuno si è salvato, che ricostruisce la sua vicenda familiare attraverso una attenta documentazione storica. Attivamente impegnato nella vita della comunità ebraica ferrarese, dall'istituzione della "Giornata della memoria" portando nelle scuole, presso i ragazzi di tutte le età, la sua testimonianza di vita. Ha scritto per Topipittori Il giorno che cambiò la mia vita nel 2009.

\section{Bodgan Bartnikowski}

Nato a Varsavia nel 1932. Viene arrestato con la madre dall'esercito russo collaborazionista durante l'insurrezione di Varsavia scoppiata il 1 agosto 1944. Dapprima condotti al campo di smistamento di Pruszków, poi ad Auschwitz. Bodgan viene registrato con il numero 192731 e rinchiuso a Birkenau, prima nella "baracca dei bambini", che si trovava in uno dei settori femminili, poi con tutti gli altri bambini di Varsavia nel settore maschile B. L'11 gennaio 1945, durante l'evacuazione del campo, una volta ricongiuntosi con la madre, viene indirizzato a Berlino Blanckenburg, un campo di lavoro che dipendeva da Sachsenhausen, dove fino al 22 aprile 1945 si occuperà della pulizia delle strade. Dopo la liberazione torna a Varsavia con la madre, unico membro della famiglia sopravvissuto oltre a lui, riprende gli studi e dopo il servizio militare lavora come giornalista. Sulla base delle proprie esperienze personali ha pubblicato molti libri sul destino dei bambini polacchi durante la guerra. In pensione, presta la sua opera gratuitamente a favore dell'Associazione dei Letterati Polacchi e dell'Associazione degli Insorti di Varsavia. 


\section{Liliana Segre}

Liliana Segre, milanese, senatrice a vita dal gennaio 2018, è nata il 10 settembre 1930. Dopo la prematura morte della mamma ha vissuto con il padre, Alberto Segre, e i nonni paterni, Giuseppe Segre e Olga Loevvy. La famiglia era laica e Liliana prese coscienza della sua "ebraicità" proprio con l'emanazione delle leggi razziali, in particolare con l'espulsione da scuola.

Quando le persecuzioni ai danni degli ebrei italiani si intensificarono, utilizzando documenti falsi, suo padre la nascose presso degli amici. Il 10 dicembre 1943 Liliana, suo padre e due cugini provarono a fuggire a Lugano, in Svizzera: i quattro furono però respinti dalle autorità del paese elvetico. Il giorno dopo furono arrestati a Selvetta di Viggiù e dopo sei giorni in carcere a Varese furono trasferiti a Como e poi a Milano, dove, tredicenne, Liliana fu detenuta per quaranta giorni al carcere di San Vittore.

Il convoglio che condusse Liliana e suo padre ad Auschwitz-Birkenau partì da quello che oggi è il "binario 21" della stazione centrale di Milano il 30 gennaio 1944. All'arrivo, sette giorni dopo la partenza, la ragazza vene separata dal padre, che non rivide mai più e che morì il 27 aprile dello stesso anno. Il 18 maggio 1944 i nonni paterni di Liliana vennero arrestati a Inveriggio, in provincia di Como e furono poi anch'essi deportati ad Auschwitz. Furono uccisi al loro arrivo, il 30 giugno 1944.

Ritenuta più grande dei suoi 13 anni, Liliana superò la selezione iniziale e ricevette il numero di matricola 75190, che le venne tatuato sull'avambraccio. Fu impiegata nel lavoro forzato presso la fabbrica di munizioni Union, che apparteneva alla Siemens, lavoro che svolse per circa un anno. Durante la sua prigionia subì altre tre selezioni. Alla fine di gennaio del 1945, dopo l'evacuazione del campo, affrontò la marcia della morte verso la Germania. Il 1 maggio 1945 l'Armata Rossa liberò il campo di Malcow, sottocampo di Ravensbruck, nel quale Liliana era stata trasferita.

Dall'Italia furono deportati ad Auschwitz 776 bambini italiani di età inferiore ai 14 anni: Liliana è tra i venticinque sopravvissuti.

Dopo il ritorno in Italia, Liliana visse con i nonni materni, di origini marchigiane, unici superstiti della sua famiglia. Nel 1948 conobbe Alfredo Belli Paci, che era stato internato per essersi rifiutato di aderire alla repubblica di Salò. Alfredo e Liliana si sono sposati nel 1951 e hanno avuto tre figli.

\section{Schede lavoro}

Liliana e Bodgan

\begin{tabular}{|l|l|l|}
\hline caratteristica & Liliana & Bodgan \\
\hline nazionalità & & \\
\hline religione & & \\
\hline
\end{tabular}




\begin{tabular}{|c|c|}
\hline \multirow{2}{*}{\begin{tabular}{|l}
$\begin{array}{l}\text { Luogo di } \\
\text { arresto }\end{array}$ \\
$\begin{array}{l}\text { E' stato/a in } \\
\text { prigione? }\end{array}$ \\
\end{tabular}} & \\
\hline & \\
\hline \multicolumn{2}{|l|}{ Causa arresto } \\
\hline \multicolumn{2}{|l|}{$\begin{array}{l}\text { Arrestato da } \\
\text { solo/a? }\end{array}$} \\
\hline \multicolumn{2}{|l|}{$\begin{array}{l}\text { Età momento } \\
\text { arresto }\end{array}$} \\
\hline \multicolumn{2}{|l|}{$\begin{array}{l}\text { Riceve } \quad \text { un } \\
\text { numero? }\end{array}$} \\
\hline $\begin{array}{l}\text { Luogo } \\
\text { internamento }\end{array}$ & \\
\hline \multicolumn{2}{|l|}{$\begin{array}{l}\text { Costretto/a } \\
\text { lavorare? }\end{array}$} \\
\hline \multicolumn{2}{|l|}{ Giornata tipo } \\
\hline \multicolumn{2}{|l|}{$\begin{array}{l}\text { Condizioni } \\
\text { baracca }\end{array}$} \\
\hline \multicolumn{2}{|l|}{$\begin{array}{l}\mathrm{Ci} \text { sono altri } \\
\text { bambini/e? }\end{array}$} \\
\hline \multicolumn{2}{|l|}{$\begin{array}{l}\text { Quando viene } \\
\text { liberato/a? }\end{array}$} \\
\hline \multicolumn{2}{|l|}{$\begin{array}{l}\text { Quando torna a } \\
\text { casa? }\end{array}$} \\
\hline \multicolumn{2}{|l|}{$\begin{array}{l}\text { Chi sopravvive } \\
\text { in famiglia? }\end{array}$} \\
\hline $\begin{array}{l}\text { Cosa succede } \\
\text { dopo la guerra? }\end{array}$ & \\
\hline
\end{tabular}

Lia, Cesare, Franco 


\begin{tabular}{|c|c|c|c|}
\hline Caratteristica & Lia & Cesare & Franco \\
\hline $\begin{array}{l}\text { Nazionalità/ città } \\
\text { di origine }\end{array}$ & & & \\
\hline $\begin{array}{lr}\text { E' } & \text { stato/a } \\
\text { cacciato } & \text { da } \\
\text { scuola? } & \end{array}$ & & & \\
\hline $\begin{array}{l}\text { Cosa ricorda del } \\
\text { non poter più } \\
\text { andare a scuola? }\end{array}$ & & & \\
\hline $\begin{array}{l}\mathrm{E}^{\prime} \text { stato/a in } \\
\text { prigione? }\end{array}$ & & & \\
\hline $\begin{array}{l}\text { E' stato/a in capo } \\
\text { di } \\
\text { concentramento? }\end{array}$ & & & \\
\hline Si è nascosto/a? & & & \\
\hline $\begin{array}{l}\text { Ha cambiato } \\
\text { identità? }\end{array}$ & & & \\
\hline $\begin{array}{l}\text { Come ha } \\
\text { imparato il nuovo } \\
\text { nome? }\end{array}$ & & & \\
\hline $\begin{array}{l}\text { Ha cambiato } \\
\text { città? }\end{array}$ & & & \\
\hline $\begin{array}{l}\text { Se ha cambiato } \\
\text { città/si } \\
\text { nascosto/a i suoi } \\
\text { genitori } \\
\text { erano? }\end{array}$ & & & \\
\hline Giornata tipo & & & \\
\hline $\begin{array}{l}\text { Quali sono gli } \\
\text { amici? }\end{array}$ & & & \\
\hline $\begin{array}{l}\mathrm{Ci} \text { sono altri } \\
\text { bambini/e? }\end{array}$ & & & \\
\hline $\begin{array}{lr}\text { Continua } & \text { a } \\
\text { studiare? } & \text { Cosa } \\
\text { pensa } & \text { dello } \\
\text { studio? } & \end{array}$ & & & \\
\hline $\begin{array}{l}\text { Quando torna a } \\
\text { casa? }\end{array}$ & & & \\
\hline
\end{tabular}




\begin{tabular}{|l|l|l|l|}
\hline $\begin{array}{l}\text { Chi sopravvive in } \\
\text { famiglia? }\end{array}$ & & & \\
\hline $\begin{array}{l}\text { Cosa succede } \\
\text { dopo la guerra? }\end{array}$ & & & \\
\hline
\end{tabular}

\section{Brani di testi utilizzati}

Si è fatta una selezione delle parti dei testi che trattano gli argomenti su cui si può operare una messa a confronto delle diverse biografie. I passi scelti consentono anche degli approfondimenti storici, che vanno valutati dall'insegnante. Molto rilievo si è dato ai sentimenti: gli autori parlano di sé e rievocano i pensieri e le emozioni che attraversavano le loro testoline. Può darsi che la stessa cosa accada con i nostri ragazzi e vale la pena approfondire il discorso.

Finzi C. M. 2009, Il giorno che ha cambiato la mia vita, Milano: Topipittori

- Alla fine dell'anno scolastico, sostengo l'esame di terza elementare, vengo promosso brillantemente e ottengo dai miei la promessa che il prossimo anno farò la quarta elementare alla scuola pubblica Umberto I. E' la scuola che frequentano i miei amici dei giardini pubblici e io desidero stare con loro anche a scuola. (pag. 25)

- E poi arriva il giorno che cambierà per sempre la mia vita. (pag. 25)

Questo breve inciso è molto interessante, perché spiega il titolo del libro, ma restituisce anche la gravità dell'entrata in vigore dei provvedimenti razziali nella vita del protagonista. Sebbene Finzi non dedichi spazio al dopoguerra, sebbene resti con tutta la sua famiglia e abbia modo di frequentare ancor degli amici, il peso di questi anni anche dopo molto tempo ci viene tutto restituito.

- E' il 3 settembre 1938 e io, un bimbetto felice di 8 anni, cammino verso il centro di Folgaria con 30 centesimi in tasca. La sera prima è arrivato il babbo e oggi ho l'incarico di andare a comprare il "Corriere Padano", giornale di Ferrara. Non lo trovo, perché nei paesi di villeggiatura, passato agosto, non arrivano più le testate delle singole città, ma solo quotidiani nazionali, così compro il "Corriere della Sera" e mi accingo a tornare a casa. Strada facendo, apro il giornale e noto un grande titolo che occupa tutta la pagina. Ormai sono grande e, purtroppo, so leggere:

INSEGNANTI e STUDENTI EBREI ESCLUSI DALLE SCUOLE GOVERNATIVE E PAREGGIATE

Capisco subito che la cosa riguarda anche me: a ottobre dovrei frequentare la quarta elementare presso la scuola pubblica Umberto I di Ferrara. Cosa significano queste parole? Non potrò più andare a scuola? Perché? Certo, sono ebreo, ma che differenza c'è tra me e gli altri bambini? E se anche ci fosse una differenza, perché non dovrei più andare a scuola? (pagg. 26-27) 
- A dire il vero, non sono mai stato uno scolaro brillante né ho avuto un amore particolare per la suola, ma davvero non mi sarà più permesso di andarci? Mi si velano gli occhi. Piango? No, forse no, ma quando raggiungo i miei a casa, mi precipito tra le braccia della mamma. I grandi mi si fanno intorno sbigottiti, frastornati, offesi. Perfino increduli. Leggono e rileggono i titoli, perfino tutti gli articoli. [...] In quei momenti ancora non lo sappiamo, ma ciò che stiamo leggendo è solo l'inizio di tutte le limitazioni vessazioni che ci saranno imposte e che dovremo subire nei giorni, nei mesi, negli anni successivi. (pag. 27)

- Con l'inizio del nuovo anno scolastico, a ottobre, riprendo a frequentare la vecchia scuola israelitica di via Vigna Tagliata che ora è diventata insufficiente ad accogliere tutti i bambini a cui è stata vietata la scuola pubblica. La mia maestra è la signora Gina Schonheit che mi ha sempre fatto un'enorme paura, grande e grossa com'è, con quel vocione. La mia è una pluriclasse che raccoglie bambini di quarta e di quinta. L'aula è la più grande della scuola. Nuovi, almeno per me, sono parecchi dei compagni, ma tutta la disposizione della classe è cambiata. ( pag. 30)

- Alcuni bambini non frequentano più la scuola. Per esempio Luciana Polacco, mia compagna di banco fino alla terza, è stata battezzata e ora, da cristiana, frequenta regolarmente la scuola pubblica. In compenso vicino a me c'è Donata Ravenna, figlia dell'ex podestà di Ferrara, che fino a giugno ha frequentato la scuola pubblica Poledrelli e che nei primi tempi è ancora più frastornata di me. La scuola ora per me diventa un incubo: se fino all'anno scorso non ho avuto molti problemi, ora a causa del terrore che mi incute la signora Gina, mi impappino sempre, anche quando saprei cosa rispondere. Mi chiedo a cosa serva studiare. (pag. 30-31)

Qui e anche oltre viene data la possibilità di spiegar le caratteristiche biologiche del razzismo italiano attraverso il tema delle conversioni al cristianesimo.

- Il governo si è posto prima di tutto il problema di definire tutto ciò che sia di "razza ebraica"... facendo gran confusione. A scuola mi hanno insegnato che esistono diverse razze di animali...fra queste esiste anche la razza umana nell'ambito della quale si possono distinguere varie etnie. Ora parrebbe che alcuni "grandi" scienziati italiani abbiano fatto una scoperta rivoluzionaria: esistono molte razze umane, alcune pure e altre impure. Il concetto di purezza della razza varia però da nazione a nazione e così per i tedeschi la razza superiore è quella ariana a cui appartengono i popoli di lingua tedesca, mentre per gli italiani è razza superiore quella latina, cui naturalmente appartiene il popolo italiano, ma non gli ebrei, che di qualunque origine siano, appartengono alla impura razza ebraica che essendo appunto impura è anche inferiore. (pag. 32)

- Secondo la nuova legge è ebreo chi nasce da due genitori ebrei anche se ormai da anni è convertito ad altra religione. E chi nasce da un matrimonio misto? Per noi ebrei è ebreo chi nasce da madre ebrea, ma la nuova legge non distingue una differenza tra i sessi. [...] Io ci capisco pochissimo in tutta questa faccenda. Per esempio, i miei cugini Rimini di Mantova [...] ora sono diventati cristiani [...] Loro quindi possono andare alla scuola pubblica, mentre noi e gli altri cugini Rimini di Milano e i Carpi d Bolzano siamo rimasti ebrei e quindi cacciati dalle scuole pubbliche (pagg. 32-33)

- Io e Nello stiamo vicini, ma presto capiamo che non faremo l'esame insieme, i nostri cognomi iniziano per F e R e ci prende lo sconforto. L'appello prosegue. Nella confusione generale, la voce del preside è coperta dal chiacchiericcio dei ragazzi e si sente a fatica. Ad un certo punto, si arriva alla lettera F, ma io non sento chiamare il mio nome; chiedo a Nello se lo ha sentito, ma la sua risposta è negativa. L'appello continua e alla fine della lettera $\mathrm{R}$ Nello Rietti non viene chiamato. Quindi, la lettura si conclude. Solo noi due non siamo stati chiamati. Siamo rimasti soli davanti al preside. «Perché non avete risposto quando è stato il vostro turno?», ci chiede. 
«Non ci ha chiamato, signor Preside», rispondiamo.

«Perché non siete stati attenti...come vi chiamate?»

«Finzi Cesare»

«Rietti Nello»

Il preside guarda nell'elenco e si accorge che, in effetti, i nostri nomi non ci sono. «Qui non ci siete. Cosa siete venuti a fare?».

Con un filo di voce, azzardo un'ipotesi: «Forse non ci siamo perché siamo ebrei?».

«Silenzio!» mi intima il Preside. Poi riguarda le carte e infine, sul retro dell'ultima pagina, in piccolo, trova i nostri nomi. (pagg. 76-77)

Non sapendo cosa fare, il Preside porta i ragazzi in un'aula, fa spostare tutti gli altri e mette loro due in fondo da soli. Quando arriva la professoressa per dettare il tema pensa che Nello e Cesare si siano messi lì in fondo per aiutarsi a vicenda. Quando anche a lei Cesare spiega che è così perché sono ebrei, nell'aula scoppia il finimondo.

- «D’altra parte, che cosa ci si può aspettare da ragazzi quotidianamente sottoposti alla propaganda fascista, da anni abituati a leggere e a vedere vignette in cui gli ebrei, in quanto razza inferiore, sono rappresentati come bestie o, peggio, diavoli?» A fatica, la professoressa riesce a far tornare un po' di silenzio; poi, dopo aver meditato un po', forse presa da pietà, dice «Spostatevi, mettetevi qui...[...]tanto non ci attaccherete la malattia».

Allora io, sciocco e ingenuo, chiedo «scusi, che malattia?»

E lei: «Come? Voi ebrei non avete la coda?»

Con Nello ci siamo poi domandati come fosse possibile che una persona laureata, e perfino tanto graziosa, potesse credere a certe frottole e arrivare a pensare davvero che avessimo le corna nascoste nei capelli e la coda nei pantaloncini... (pag. 78).

- Così papà, che nel 1915 è scappato di casa per arruolarsi nell'esercito italiano e combattere per l'unità d'Italia, non sarebbe più un italiano degno di tale nome solo perché appartiene a una religione diversa? Cosa c'entra la religione con la cittadinanza? (pag. 27)

- Dato che papà ha fatto la Grande Guerra come volontario ed è anche stato ferito, ha ricevuto la qualifica di "discriminato" sebbene si tratti sempre di un italiano di razza inferiore. Grazie a questo riconoscimento possiamo fruire di alcuni vantaggi come: possedere una radio, agli altri ebrei confiscata; tenere una domestica cristiana a servizio; frequentare alberghi benché di categoria inferiore. (pag. 33)

- Un pomeriggio, vado al parco Massari con Manlio e con la mamma. Appena arrivato vedo i miei amici che giocano nel prato. Mentre la mamma si siede su una panchina una signora, amica di mia mamma che alcune volte è stata a casa nostra e nella cui bella abitazione siamo andati spesso, improvvisamente s'alza da una panchina vicina, chiama suo figlio e si allontana senza neppure salutare mia madre. Seguono il suo esempio altre signore che, chiamati i figli, se ne vanno. Io rimango solo. Allora anche la mamma, facendo finta di niente, si alza, mi chiama, poi si alza dalla panchina e ci porta a fare un lungo giro per i viali. Non capisco subito il significato di questo episodio perché i miei genitori fanno di tutto per non farmi vivere il dramma della discriminazione. Tuttavia, in pochi giorni lo collego con i rapidi cambiamenti che stanno interessando a nostra vita. Per molto temo, il parco Massari non sarà più meta delle nostre passeggiate. (pag. 28)

- E poi è venuto il settembre 1943

Degli amici dicono allo zio di Cesare che sono riusciti ad avere nuovi documenti, senza la scritta razza ebraica, con nuovi nomi e identità e che devono quindi lasciare il posto dove vivono, perché tutti li conoscono. Lo zio capisce immediatamente l'importanza di questa notizia. Anche le nostre famiglie devono riuscire a ottenere nuovi documenti. (pag. 106)

- Ma non si tratta solo di preparare tredici carte di identità e tredici carte annonarie con nomi di fantasia. I nuovi cognomi devono essere abbastanza simili ai nostri perché per noi sia 
semplice impararli e scriverli, quando firmiamo. In più, è necessario modificare anche il luogo di provenienza, se figurassimo come provenienti dalle zone già liberate dagli anglo americani sarebbe praticamente impossibile da parte delle autorità fare un controllo. Ma nessuno di noi ha un accento dell'Italia meridionale, per questo bisogna trovare una zona di provenienza dalla Lombardia. [...] A giorni, io non sarò più Cesare Finzi, ma Cesare Franzi, non abiterò più a Ferrara, ma a Milano, in viale degli Abruzzi 55 (la casa è andata distrutta dai bombardamenti e li sotto risultano essere rimasti anche tutti i nostri documenti), (pag. 107)

- Credete sia facile inventarsi una nuova identità e una nuova vita? Provate un po' voi! Non si tratta di un gioco, c'è di mezzo la vita di tutti. Come spiegarlo ai più piccoli, si chiedono gli adulti, specie Silvana e Graziana che da poco hanno imparato a dire i loro nomi e indirizzi veri? Ecco allora che in casa è tutto un susseguirsi di presentazioni e di risposte, di firme e controfirme...Un caos! (pag. 107)

- Ora che siamo in possesso dei documenti dobbiamo scappare da Gabicce dove tutti ci conoscono con i nostri veri nomi. Si pone il problema di dove andare. Spostarsi in treno è sempre più difficile e pericoloso a causa dei bombardamenti. (pag. 109)

Inoltre spiega Cesare trovare una sistemazione per 13 persone nei dintorni, dove tutti si rifugiano proprio per sfuggire alle bombe, è difficile. Il libro prosegue con il racconto delle fughe, della ricerca di rifugi. Si arriva fino al 1945.

- E, finalmente, il 15 ottobre riaprono le scuole. Per la prima volta, posso presentarmi all'appello come tutti gli altri studenti. Con il batticuore, salgo le scale del Liceo Scientifico “A. Roiti". «Cosa sarà di me?» mi chiedo. «Come mi accoglieranno i nuovi compagni?». Sono ragazzi e ragazze che per anni, fino a pochi mesi fa, sono stati indotti dalla propaganda del regime a considerare gli ebrei esseri inferiori, nemici da denunciare, quando non da eliminare...Come potranno accettare di vivere e condividere tante ore di studio con me? Come potrò dire loro che sono ebreo? Qualcuno mi accetterà per quello che sono? Nell'atrio del Liceo il preside ci saluta e comincia a fare l'appello, e alora vengo assalito dal ricordo di un altro appello: quello, interminabile, avvenuto due anni prima. Penso al mio grande amico Nello Rietti che ora non è con me, perché è stato ammazzato da quei maledetti...Uno dopo l'altro, il preside legge i nomi dei ragazzi. Mi coglie il terrore che non chiami il mio. Invece, quando arriva alla terza A, legge: «Esposito, Felletti, Finzi...»

Ci sono. (pag. 189)

\section{Levi L, Trilogia della memoria- Il francese, ebook}

- Papà è disoccupato e questo vuol dire che non esce più la mattina per andare in ufficio. Qualche volta ci accompagna ancora a scuola, ma prima andare con lui era un'emozione, adesso è un gioco fiacco.

\section{Levi L, Trilogia della memoria-Roma, ebook}

- Papà ora ha un lavoro. Un signore gli ha detto che aveva bisogno di lui nel suo ufficio, solo che il suo ufficio è a Milano e così noi dobbiamo andare ad abitare in quella città. Partiamo tutti, anche la donna, Maria. 
- Ma quel signore che ha dato lavoro a mio papà, poco dopo cambia idea: il lavoro c'è sempre, ma non è più a Milano, è a Roma. [...] Cambiare ancora la scuola questa volta non è così facile. Siamo a metà dell'anno scolastico e sembra che loro abbiano studiato tutto diverso, più avanti, più indietro...

\section{Levi L. 1994, Una bambina e basta, Roma: Edizioni E/O}

- Mio padre sa già tutto, ha compreso tutto, ma resta immobile. Non è più mio padre, ma l'eterno uomo ebreo che si ferma smarrito quando quello che da tanto si portava dentro, che la sua mente aveva disegnato in ogni più minuta piega è li, improvvisamente di fronte a lui...non sa battersi per sopravvivere perché quando suo padre, suo nonno, il suo bisnonno hanno lottato, hanno via via consumato anche le sue forze (pag. 52)

- Le madri ebree, no, sono tigri, leonesse, contendono alla vita ogni boccone, rubano ogni centimetro. Loro devono difendere i figli: per questo non hanno spazio per libri e sinagoghe. (pag. 52)

- $\quad$ siamo gli unici ad avere la donna di servizio perché agli ebrei è proibito. Ma mio padre è discriminato, così c'è scritto sui fogli che ci hanno dato, ma questo lo sapevamo anche prima, perché è figlio di un ufficiale caduto in guerra, quella del '15 -'18. (pag. 30)

- Comunque questo nostri essere discriminati era l'ultimo scampolo di privilegio, ma dopo un po' il mantello era diventato stretto. Veniva a casa uno, gentile, della questura e diceva: "Non si può". Un giorno ci aveva portato via la radio, sempre dicendo "non si può", ma poi, forse perché eravamo ebrei speciali, o forse perché bastava insistere, la radio finiva per tornare a casa. Come Maria. Anche per lei ogni tanto quello della questura diceva "Non è possibile", ma poi tornava tutto normale. (pag. 31)

- Un'altra cosa che ha un "non si può" è andare in villeggiatura, ma lì per lì non serve il mantello del solito nonno di Caporetto. C'è un'altra nonna, la mamma di mia mamma. La nonna prende una casa al mare e noi, solo noi bambine, andiamo da lei. Allora è chiaro che la nonna non è ebrea. (pag. 32)

- Ma questa volta l'uomo della questura è definitivo: "non può più stare a casa di ebrei, se ne deve andare". Andarsene? Maria? E' come dire che non potrà più piovere. Parlano $\mathrm{e}$ parlano, poi i miei decidono di chiedere alla vicina di casa se vuole fare finta di avere Maria al suo servizio. Maria dormirebbe lì da loro e per farsi accettare potrebbe verso sera aiutare un po' lavare i piatti e cose del genere e per il resto rimanere sempre da noi come prima. I vicini accettano, evviva. Maria resterà con noi, come appunto è sempre stata. (pagg.33 -34)

- Provo un moto strano quando la vado a chiamare nell'altra casa e lei apre la porta con un grembiule che non conosco: "ho da fare...hanno ospiti". [...] Poi c'è sempre qualcosa di nuovo.[...] La signora della porta accanto è bionda, un po' monumentale, decisamente ottusa. Lei è la signora e quella è la sua donna, anche se la paghiamo noi. (pag. 34)

- Hanno pensato di allontanare noi bambine. Allontanarci? Per andare dove? Beh, la mamma ha trovato un grande convento, un educandato appena fuori città. Le suore conoscono d tanto tempo la direttrice della scuola ebraica e sono così d'accordo di prenderci da loro: andremo noi tre, più la figlia e la nipote dell'amica di mamma e forse qualcun'altra. (pag. 53)

- Mi hanno cambiato nome e naturalmente cognome. Le mie sorelle forse non sono più le mie sorelle, dato che il loro cognome è diverso. C'è stato, prima, un momento in cui erano tutti preoccupati. L'ho capito perché mia madre, ancora una volta, non sui curava che io ascoltassi i loro discorsi. Sembra che il Vaticano abbia avvertito che forse i tedeschi entreranno nel convento a cercare la gente come noi e gli altri che si nascondono. (pag. 74) 
- Per noi le suore hanno avuto un'idea. Molte collegiali per l'estate erano andate a casa per le vacanze scolastiche, ma, dato che abitavano al sud, sono rimaste bloccate là dalla guerra. Qui nel convento sono rimaste molte delle loro cose, qualche vestito negli armadietti di ferro, libri e quaderni e quello che è più importante tutti i loro documenti. E' così semplice, basta scegliere l'età giusta e noi diventiamo loro. A me capita "Maria Cristina Cataldi". Non Elena o Lucia, ma Maria Cristina: è il massimo. Con un nome così, c'è da scontare a vita anni di complesso ebraico. (pag. 75)

- Ora però questi nuovi nomi li dobbiamo imparare a dirli bene, senza dimenticare la città dove dobbiamo fare finta di essere nate. Specie per 1 più piccole, è tutta una baraonda. $\mathrm{Ci}$ esercitiamo per ore nei corridoi come se stessimo provando uno dei nostri spettacoli di teatro. Il più difficile è riuscire a girarsi subito quando qualcuno ti chiama all'improvviso da lontano con il tuo nome finto. Diventiamo bravissime, si sa, nel gioco siamo allenate a vincere. Resta il problema di Rossana. Nessuna bambina così piccola è mai entrata in convento e tanto meno ha lasciato i suoi documenti. Le suore ci pensano molto e poi decidono: diranno che l'hanno trovata sotto le macerie di una casa bombardata e che l'hanno raccolta senza sapere assolutamente chi è. Ci mettiamo tutte a parlare a Rossana «ti hanno trovata sotto le macerie, hai capito?». Lei ha capito benissimo. A chi le domanda come si chiama, grida trionfante «Spepetto sottomacerie». "Sottomacerie" è il suo nuovo cognome. (pag. 76)

- "Maria Cristina Cataldi", "Maria Cristina Cataldi". Continuo ad aggrapparmi al mio nome falso, il solo salvagente che ho, mormorandolo e rimormorandolo come una personale orazione... Cosa vogliono i tedeschi? Che cosa gli sta dicendo la portinaia? C'è il sangue dentro di me, ma al cervello non arriva: ho solo sensazioni, non pensieri e so che non potrei pronunciare nemmeno una sillaba. (pag. 84)

- Ma cosa ci sarà da fare per diventare cristiana? Poi un giorno tutto si rischiara di una luce improvvisa: chiederò a suor Maria Speranza. [...] passo un giorno e una notte a tremare e poi all'improvviso la blocco dopo la lezione a scuola. Le parlo concitata e sconnessa, le dico che sono state le sue parole, solo le sue fra le tante che tutti mi buttavano addosso, ad affondare in me. (pag. 87)

- Le suore continuano a dirmi «vieni, vieni» e io non ho la minima idea di dove mi vogliono trascinare . Attorno è già confusione. Prima non ero andata a mettere i libri a posto dopo la scuola, poi, molto tardi, sono arrivata accompagnata dalla monaca insegnante...cosa sta succedendo? (pag. 89)

- Siamo in chiesa. E' tutto gelido e nero tranne il lacrimoso candelotto sotto Gesù Cristo. L'odore di incenso mi è insopportabile e cerco di respirare solo con la bocca. [...] Non c'è musica, né cori né preghiere solo i nostri passi che fanno un rumoroso irriverente TOC sul pavimento di marmo. Mi afferra l'orrore e una specie di vortice di panico. Cosa sto facendo qui io, la bambina della scuola ebraica, che fino a ieri cantava a squarciagola con tutte le altre "Maoz tzur" quando sfavillavano le luci di Chanuccà? Poi mia sorella comincia a singhiozzare. Piange, piange, piange e mentre il mio sguardo finge ancora il fastidio, tutto il mio essere vola verso di lei. (pag. 90)

- Siamo tutte impalate..ora suor Concetta apre il portale grande e così, senza che nessuno abbia deciso, ci troviamo subito all'aperto sotto il portico. Resto impietrita. Lì, di fronte a noi, l'immagine della furia, c'è mia madre. Il nostro segreto è durato nemmeno dieci minuti, le ragazze lo hanno sentito nell'aria, qualcuna è corsa a chiamarla, non so. Mia madre non è mia madre, i sui capelli sono serpenti, i suoi occhi scintille di fuoco, la sua voce, come Sansone, fa tremare le colonne del chiostro. Mia madre grida e accusa. Forse sta per sbranare le piccole suore, non le viene in mente che loro ci possono buttare fuori, in mezzo al pericolo, anche se a noi lo dice ogni giorno. Suor Speranza e suor Concetta, investite dalla sua violenza, vacillano, tremano anche loro, come prima le colonne del chiostro. (pag. 9091) 
Note:

[1] Ad esempio possono essere visionati:

https://www.youtube.com/watch?v=w1bKp3qRN7w e

https://www.youtube.com/watch?v=sVTtoXeQgZ0

[2] Si consigliano tra gli altri: Liliana Segre, Enrico Mentana, La memoria rende liberi. La vita interrotta di una bambina nella Shoah, Rizzoli, 2015; Liliana Segre, Scolpitelo nel vostro cuore. Dal Binario 21 ad Auschwitz e ritorno: un viaggio nella memoria, Milano, Piemme, 2018; Liliana Segre, Daniela Palumbo, Fino a quando la mia stella brillerà, Milano, Piemme 2015.

[3] Bodgan Bartnikowski è nato a Varsavia nel 1932. Viene arrestato con la madre dall'esercito russo collaborazionista durante l'insurrezione di Varsavia scoppiata il 1 agosto 1944. Dapprima condotti al campo di smistamento di Pruszków, poi ad Auschwitz. Bodgan viene registrato con il numero 192731 e rinchiuso a Birkenau, prima nella "baracca dei bambini", che si trovava in uno dei settori femminili, poi con tutti gli altri bambini di Varsavia nel settore maschile B. L'11 gennaio 1945, durante l'evacuazione del campo, una volta ricongiuntosi con la madre, viene indirizzato a Berlino Blanckenburg, un campo di lavoro che dipendeva da Sachsenhausen, dove fino al 22 aprile 1945 si occuperà della pulizia delle strade. Dopo la liberazione torna a Varsavia con la madre, unico membro della famiglia sopravvissuto oltre a lui, riprende gli studi e dopo il servizio militare lavora come giornalista. Sulla base delle proprie esperienze personali ha pubblicato molti libri sul destino dei bambini polacchi durante la guerra. In pensione, presta la sua opera gratuitamente a favore dell'Associazione dei Letterati Polacchi e dell'Associazione degli Insorti di Varsavia.

Nel 2017, 2018, 2019, in corrispondenza del giorno della sua uscita dal campo di Auschwitz è stato a Novara accompagnato da Jadwiga Pinderska-Lech, direttrice della Casa Editrice del Museum Auschwitz-Birkenau per incontrare gli studenti delle scuole cittadine e i ragazzi partecipanti al progetto Promemoria Auschwitz.

Si veda la proposta di scheda "Liliana e Bodgan"

[5] In inglese "hidden Children", in fracese "enfants chachés", "bambini nascosti" è il termine utilizzato in italiano per indicare $\mathrm{i}$ bambini, prevalentemente ebrei, che sono stati nascosti durante l'Olocausto in modo da essere salvati. Talvolta i bambini erano allontanati dalla famiglia e collocati presso conoscenti, presso strutture religiose, ecc. Talvolta i gruppi familiari si nascondevano tutti insieme. Si tratta di una modalità di salvamento molto articolata non ancora del tutto studiata, soprattutto per i casi italiani.

[6] La mostra, oggi esistente in tre copie che hanno continuato a circolare dal 2008, si intitola "17 novembre 1938 lo stato italiano emana le leggi razziali"

[7] Si vedano a tal proposito W A. Wieviorka, Les enfants caches sont-ils un objet d'histoire?, in D. Bailly (cordonné par), Enfants cachés. Analyses et débats, Actes de la Journée d'Étude du 18 novembre 2005, L'Harmattan, Paris 2005 e la tesi di dottorato presso l'Università di Verona di Paolo Tagini. A pag. 29 in nota Tagini riporta un passaggio delle pagg. 44-45 del testo sopra citato «Alors que le sauvetage des Juifs en France, lui, est bien un sujet d'histoire, pour 
lequel, compte tenu du manque de traces écrites, l'enquête orale est indispensable, en revanche. La constitution de ceux qui ont été cachés comme sujet collectif d'histoire me semble acrobatique». [allorché il salvamento degli ebrei in Francia è a tutti gli effetti un soggetto con dignità storica per il quale, tenuto conto della mancanza di fonti scritte, la ricerca orale è indispensabile...considerare coloro che sono stati nascosti come un soggetto di storia mi sembra acrobatico] Tagini aggiunge poi questo commento: «Secondo Wieviorka, les enfants cachés per lo storico sono simili a granelli di sabbia che scivolano via dal palmo della sua mano. Semmai è l'analisi della memoria degli enfants cachés, osservata nella sua essenza, nella sua evoluzione nel tempo, con i suoi oblii e le sue limpidezze, il vero oggetto di ricerca? Ogni gruppo vittima della storia rivendica ormai, a detta di Wieviorka, il riconoscimento da parte della ricerca storica delle proprie sofferenze, tanto da protestare vigorosamente quando il lavoro dello storico non restituisce l'immagine che essi hanno di loro stessi. L'analisi del caso italiano contraddice, senza dubbio, quest'ultima affermazione della studiosa francese: come si avrà modo di vedere, in Italia, infatti, gli ebrei ex bambini nascosti né hanno mai rivendicato un'appartenenza di gruppo, né un proprio specifico riconoscimento storiografico».

Paolo Tagini, pag. 22 «Il racconto e il ricordo del genocidio ebraico, così grande e terribile, è stato considerato per molto tempo dalla società occidentale più un problema che una risorsa. Colui che, suo malgrado, è passato attraverso l'esperienza della deportazione e del campo di sterminio ha visto crescere nei suoi riguardi l'attenzione dei media, delle istituzioni delle varie categorie sociali: l'interesse suscitato, che sia stato autentico o strumentalizzato, si è concentrato su questa speciale tipologia di testimone, lasciando nell'ombra coloro che invece hanno vissuto lo stesso tempo e potenzialmente gli stessi rischi, ma che infine hanno avuto un destino molto diverso da quello che fu riservato a tutti gli ebrei deportati». Pag. 24 «Quando, dopo la liberazione, molti hidden children cominciarono a udire notizie di camere a gas, di forni crematori, di violenze, di uccisioni indiscriminate, quando intravidero i numeri tatuati sulla pelle dei sopravvissuti e ne chiesero spiegazione, rimasero scioccati. Da quel momento essi intuirono che misurare le proprie esperienze con quelle dei correligionari ritornati dai campi della morte nazisti non sarebbe stato possibile, come non sarebbe stato pensabile - alla pari di quanto avvertito da Friedländer - immaginarsi (e quindi riconoscersi) al centro della tragedia; per questo motivo, molti non si sentirono nemmeno in diritto di "reclamare" alcuna compassione e, se lo fecero, rivelarono con molta fatica le loro storie di clandestini».

[8] Affermazione dello stesso Debenedetti in diversi incontri di testimonianza

[9] Affermazione dello stesso Debenedetti in diversi incontri di testimonianza

[10] Medaglietta a forma di stella di david che viene data alla nascita ai bambini ebrei 